\title{
Actividad física en adolescentes de cinco ciudades colombianas: resultados de la Encuesta Mundial de Salud a Escolares
}

\author{
Physical activity in adolescents of five Colombian cities: Results of \\ the Global Youth Health Survey
}

\author{
Marion Piñeros y Constanza Pardo \\ Instituto Nacional de Cancerología. Bogotá, Colombia. mpineros@cancer.gov.co \\ Recibido 24 Marzo 2010/Enviado para Modificación 20 Octubre 2010/Aceptado 1 Diciembre 2010
}

\section{RESUMEN}

Objetivo Las enfermedades crónicas representan una carga considerable de la enfermedad en Colombia. La promoción de actividad física constituye una de las intervenciones más exitosas para su control. La vigilancia de los niveles de actividad física en escolares, permitirá el diseño de programas para su promoción así como su monitoreo y evaluación. Estimar la prevalencia de actividad física global, actividad física para el transporte, sedentarismo y clases de educación física en jóvenes escolares de cinco ciudades de Colombia.

Materiales y métodos Se implementó la Encuesta Mundial de Salud a Escolares en 2007, en colegios públicos y privados de Bogotá, Bucaramanga, Cali, Manizales y Valledupar. Se hizo un muestreo bietápico por conglomerados, de colegios y clases cuyos resultados son representativos por ciudades para los jóvenes escolares. La encuesta fue anónima, voluntaria y auto-diligenciada por los estudiantes. El análisis estadístico se realizó en los Centros para el Control y Prevención de Enfermedades (CDC).

Resultados Los niveles de actividad física recomendada reportados por jóvenes de 13 a 15 años estuvieron entre $9 \%$ y 19,8 \% en las cinco ciudades, siendo más altos en hombres. $50,3 \%$ de los jóvenes reportaron no realizar actividad física para su transporte al colegio y 50,3\% indicaron que pasaban tres horas o más diarias frente al televisor o pantalla de computador.

Conclusión Los bajos niveles de actividad física en adolescentes de las cinco ciudades colombianas indican la necesidad de intervenciones en distintos niveles y nuevo monitoreo.

Palabras Clave: Vigilancia epidemiológica, actividad motora, adolescente, Colombia (fuente: DeCS, BIREME).

\section{ABSTRACT}

Objectives In Colombia, chronic diseases represent a significant health burden. The promotion of physical activity has demonstrated effectiveness in terms of their control. The surveillance of physical activity levels in different age groups permits 
the design of specific interventions, monitoring and evaluation. To estimate the prevalence of global physical activity, sedentarism, physical activity for transportation and physical activity at school, among adolescent students in five Colombian cities.

Materials and methods The Global School-based Health Survey was implemented in public and private schools of five Colombian cities: Bogotá, Bucaramanga, Cali, Manizales y Valledupar. Students were selected through a multi-stage sampling procedure. The survey was anonymous, voluntary and self-processed by students. Statistical analysis was performed at the CDC. The results are representative for adolescent students of each city.

Results The reported recommended levels of physical activity in students from 13 to 15 years ranged from $9 \%$ to $19.8 \%$ in the five cities, being constantly higher among males. Overall, $50.3 \%$ of the students reported not being physically active for transportation to school. $50.3 \%$ of the students played video or computer games or used a computer for 3 or more hours a day.

Conclusion The results indicate low levels of physical activity among students of the five main cities in Colombia, pointing towards the need of interventions at different levels and the need for continuing surveillance.

Key Words: Surveillance, physical activity, adolescent, Colombia (source: MeSH, NLM).

$\mathrm{L}$

as enfermedades crónicas no transmisibles son la principal causa de muerte en el mundo con una carga significativa en países en vías de (1). Para su prevención y control se han implementado intervenciones orientadas a reducir la prevalencia de los principales factores de riesgo que comparten la mayoría de ellas. Estas intervenciones buscan reducir la prevalencia del consumo de tabaco y alcohol así como incrementar la realización de actividad física y el consumo de frutas y verduras. En cuanto a la actividad física existe evidencia definitiva y suficiente en términos de la efectividad de la misma para la prevención de enfermedades cardiovasculares, diabetes, obesidad, hipertensión, osteoporosis y cáncer (2). Los cánceres en los cuales hay evidencia del papel protector de la actividad física son cáncer de colon y cáncer de mama.

La promoción de hábitos saludables conlleva necesariamente una orientación sobre la frecuencia e intensidad de su realización para obtener los efectos deseables. En este sentido, parece haber un consenso de que lo más recomendable es practicar actividad física de forma regular al menos 3 días por semana y de ser posible todos los días para completar al menos 150 minutos semanales.

Por las características propias de la edad, las recomendaciones en jóvenes son distintas a las de adultos. Para niños y adolescentes las recomendaciones 
europeas y australianas establecen que lo ideal es realizar al menos una hora diaria de actividad física de intensidad moderada (3). A su vez, la SociedadAmericana contra el Cáncer recomienda para esta población la práctica de 60 minutos diarios al menos cinco días en la semana (4).

Los adolescentes y particularmente aquellos que son estudiantes, conforman un grupo poblacional de gran interés para la promoción de actividad física por varias razones. Así como la obesidad en la niñez se relaciona con la obesidad en la vida adulta (5), también se ha encontrado que un mayor nivel de actividad física en una edad temprana se asocia con una mayor práctica de la misma en la edad adulta (6). Adicionalmente, se ha determinado que los comportamientos adquiridos en esta edad suelen mantenerse (7) y se cuenta con evidencia de intervenciones exitosas en el ámbito escolar para incrementar la actividad física (8).

La Encuesta Mundial de Salud a Escolares (EMSE, por sus siglas en español) es una herramienta que permite contar con información sobre diversos aspectos de la salud en jóvenes, entre las que se encuentra la medición de actividad física. Su aplicación permite contar con información de base para establecer programas de salud escolar, monitorear tendencias y comparar las prevalencias de factores de riesgo y protectores ente ciudades y países.

La realización de la EMSE ha sido promovida a nivel mundial por la Organización Mundial de la Salud (OMS) con el apoyo de los Centros para el Control y la Prevención de Enfermedades (CDC) en los Estados Unidos. En la región de las Américas y el Caribe ha sido aplicada y finalizada en veinte países (9) y en Colombia se aplicó por primera vez en 2007, en cinco ciudades para evaluar la prevalencia de distintos factores de riesgo y protectores para enfermedades crónicas.

En este artículo se presentan los resultados relacionados con los niveles de actividad física en los estudiantes de 13 a 15 años en Bogotá, Bucaramanga, Cali, Manizales y Valledupar. Estos resultados tienen particular relevancia para el país pues en Colombia, se cuenta con muy poca información sobre este tema en adolescentes.

\section{MATERIALES Y MÉTODOS}

La Encuesta Mundial de Salud a Escolares fue implementada por el Instituto Nacional de Cancerología con la colaboración técnica de la OMS, la 
Organización Panamericana de la Salud (OPS) y los Centros para el Control y Prevención de Enfermedades (CDC).

El protocolo de la encuesta fue aprobado por el Comité de Ética en Investigaciones del Instituto Nacional de Cancerología y la implementación se realizó en el segundo semestre de 2007.

La EMSE se aplicó en colegios públicos y privados de cinco ciudades de Colombia (Bogotá, Bucaramanga, Cali, Manizales y Valledupar), en los grados 7 a 10, de manera que incluyera los estudiantes de 13 a 15 años. Con excepción de Bogotá, las ciudades se seleccionaron por tener un registro de cáncer que permita en un futuro el desarrollo de intervenciones en salud pública y la medición de incidencia de cánceres relacionados.

El cuestionario de la EMSE aplicado en Colombia contenía 81 preguntas de distintos temas (Aspectos demográficos; hábitos alimentarios; actividad física; consumo de tabaco; consumo de alcohol; relaciones sexuales). En relación con la actividad física se indagó sobre realización de actividad física global, actividad física para el transporte al colegio, actividad física como parte del currículo escolar y sedentarismo (tiempo sentados viendo televisión o jugando en computador).

La EMSE se aplicó en los colegios de manera simultánea con la Encuesta Mundial de Tabaquismo (EMTAJ, por sus siglas en español). Los padres de familia fueron notificados antes de la encuesta y podían negarse a la participación de sus hijos en la misma. Los procedimientos de la encuesta fueron diseñados para proteger la intimidad de los alumnos, permitiendo una participación anónima y voluntaria. Los alumnos completaron el cuestionario durante una hora normal de clase y registraron sus respuestas directamente en una hoja de respuestas apta para su escaneo posterior.

\section{Muestra}

El cálculo de la muestra y las estimaciones se realizaron con base en la metodología estandarizada por los Centers for Disease Control and Prevention (10). El marco muestral fue de 2228 colegios públicos (36\%) y privados (64\%) existentes a 2006, para un total de 663703 estudiantes en los grados 7 a 10, con una distribución mayor en los públicos $(62 \%)$ que en los privados $(38 \%)$. Se calculó una muestra representativa e independiente para cada ciudad basada en el total de colegios públicos y privados mencionados. 
El muestreo fue bietápico por conglomerados. En la primera etapa de muestreo se realizó la selección de los colegios con una probabilidad proporcional al número de estudiantes matriculados en los grados 7 a 10. La segunda etapa del muestreo consistió en una selección aleatoria de clases (cursos) en cada colegio. Todas las clases se incluyeron en el marco de la muestra y todos los alumnos de cada clase muestreada fueron elegibles para participar en la encuesta EMSE.

Fueron seleccionados un total de 191 colegios entre públicos y privados. Para las ciudades de Bogotá y Cali se seleccionaron 50 colegios en cada una, para Valledupar 31 y para Bucaramanga y Manizales 30 en cada ciudad.

Procesamiento y análisis de datos

Las hojas de respuesta diligenciadas se remitieron a los CDC para su procesamiento.

Se calcularon tres índices de respuesta: 1. la tasa de respuesta escolar, que se calculó con el número de escuelas participantes sobre el número de clases seleccionadas; 2 . la respuesta estudiantil que se calculó con el número de estudiantes participantes sobre el total de estudiantes matriculados, y; 3 . la respuesta total que se calculó como el producto entre la tasa de respuesta escolar y la respuesta estudiantil.

Por cada alumno se aplicó un factor de ponderación que se calculó como el inverso de la probabilidad de selección para cada etapa y se ajustó por la falta de respuesta para cada colegio, clase y estudiante. La generación de los factores de ponderación y el cálculo de los estimadores con los intervalos de confianza respectivos se hicieron con el programa SUDAAN (11). Esta ponderación permite aplicar los resultados a toda la población de estudiantes por ciudad. En Bogotá, los resultados además tienen representatividad por tipo de colegio, para colegios oficiales y privados.

Se consideraron las diferencias estadísticamente significativas con una $\mathrm{p}<0,05$.

La actividad física global se analizó con dos mediciones: \% de jóvenes que realizan actividad física al menos 60 minutos durante 7 días de la semana y \% de jóvenes que realizaron actividad física al menos 60 minutos durante menos de cinco días de la semana. La actividad física para el transporte al colegio se expresó como la no realización de la misma para el transporte. 


\section{RESULTADOS}

Índices de respuesta y características de los participantes

En total participaron 9907 estudiantes, de los que $6833(68,1 \%)$ estaban en el rango de 13 a 15 años de edad. El porcentaje de mujeres que completaron la encuesta $(54,8 \%)$ fue mayor que el de hombres (Tabla 1). La Tabla 1 muestra el número de participantes en cada ciudad, discriminado por sexo, edad y clases.

La tasa de respuesta escolar en las cinco ciudades fue de $93 \%$. Esta tasa se vio afectada por colegios que no quisieron participar (11) pero también porque al aplicar ambas encuestas en cada colegio, hubo colegios en los que no se aplicó la EMSE y solamente la EMTAJ. La tasa de respuesta estudiantil varió entre $86 \%$ y $91 \%$, con la tasa más baja de respuesta en la ciudad de Cali (Tabla 1).

Tabla 1. Participación escolar y características de los alumnos en cada ciudad, Encuesta Mundial de Salud a Escolares, Colombia 2007

\begin{tabular}{|c|c|c|c|c|c|c|}
\hline Características & Bogotá & Bucaramanga & Cali & Manizales & Valledupar & Total \\
\hline \multicolumn{7}{|l|}{ Indices de participación } \\
\hline Número de colegios & 46 & 28 & 45 & 28 & 30 & 177 \\
\hline Respuesta escolar (\%) & 92 & 93 & 90 & 93 & 97 & 93 \\
\hline Respuesta estudiantil (\%) & 90 & 86 & 86 & 91 & 88 & 88,2 \\
\hline Respuesta total (\%) & 83 & 81 & 77 & 85 & 86 & 82,4 \\
\hline Total alumnos participantes & 1.737 & 2.093 & 1.945 & 2.037 & 2.095 & 9.907 \\
\hline Hombres (\%) & 45,5 & 47,1 & 44,0 & 45,4 & 44,0 & 45,2 \\
\hline Mujeres $(\%)$ & 54,5 & 52,9 & 56,0 & 54,6 & 56,0 & 54,8 \\
\hline \multicolumn{7}{|l|}{ Edad } \\
\hline 12 años o menos (\%) & 11,0 & 10,7 & 22,6 & 8,1 & 13,5 & 13,2 \\
\hline 13 a 15 años $(\%)$ & 68,2 & 72,7 & 64,9 & 66,4 & 68,5 & 68,1 \\
\hline 16 años o más $(\%)$ & 20,8 & 16,6 & 12,6 & 25,5 & 18,1 & 18,7 \\
\hline \multicolumn{7}{|l|}{ Grados (clases) } \\
\hline $7^{\circ}(\%)$ & 28,0 & 26,8 & 29,4 & 27,7 & 30,0 & 28,4 \\
\hline $8^{\circ}(\%)$ & 26,0 & 25,4 & 26,4 & 26,8 & 25,6 & 26,0 \\
\hline $9^{\circ}(\%)$ & 24,6 & 25,1 & 23,8 & 23,8 & 24,3 & 24,3 \\
\hline $10^{\circ}(\%)$ & 21,4 & 22,7 & 20,4 & 21,7 & 20,1 & 21,3 \\
\hline \multicolumn{7}{|l|}{ Participantes de 13 a 15} \\
\hline años & 1.255 & 1.510 & 1.299 & 1.367 & 1.402 & 6.833 \\
\hline Hombres & 550 & 701 & 568 & 608 & 592 & 3.019 \\
\hline Mujeres & 702 & 806 & 731 & 755 & 807 & 3.801 \\
\hline
\end{tabular}

Actividad física y sedentarismo

Actividad física global. La prevalencia de actividad física recomendada (60 minutos diarios durante siete días de la semana) en jóvenes de 13 a 15 años de 
las cinco ciudades varió entre $9,1 \%$ y $19,8 \%$, con un promedio de $15 \%$. La ciudad con mayor proporción de alumnos que reportaron niveles de actividad física recomendada fue Cali $(17,1 \%)$; la ciudad con menor prevalencia de actividad física fue Manizales (12,1\%), aunque las diferencias no fueron significativas. En los hombres, la mayor prevalencia de actividad física estuvo en Bucaramanga y en las mujeres, en Cali. En todas las ciudades las mujeres reportaron menores niveles de actividad física, con diferencias significativas entre hombres y mujeres en Manizales y Valledupar (Tabla 2).

Tabla 2. Prevalencia de actividad física en estudiantes de 13 a 15 años de edad por sexo, según ciudad, Colombia, EMSE 2007

\begin{tabular}{|c|c|c|c|}
\hline \multirow{2}{*}{ Actividad física-Variables } & \multicolumn{3}{|c|}{ Prevalencia \% (IC 95\%) } \\
\hline & Hombres & Mujeres & Total \\
\hline \multicolumn{4}{|c|}{ Actividad física al menos 60 minutos diarios durante 7 dias a la semana } \\
\hline Bogotá, D.C. & $18,1(14,5-21,8)$ & $12,8(8,4-17,1)$ & $15,1(12,1-18,0)(n=1.253)$ \\
\hline Bucaramanga & $19,8(16,2-23,4)$ & $13,9(11,2-16,6)$ & $16,6(14,3-19,0)(n=1.496)$ \\
\hline Cali & $19,7(15,5-24,0)$ & $15,0(10,8-19,1)$ & $17,1(13,6-20,5)(n=1.283)$ \\
\hline Manizales & $16,0(12,8-19,2)$ & $9,1(7,0-11,2)$ & $12,1(10,3-13,9)(n=1.355)$ \\
\hline Valledupar & $17,8(14,6-20,9)$ & $11,7(9,8-13,6)$ & $14,3(12,5-16,2)(n=1.389)$ \\
\hline \multicolumn{4}{|c|}{ Actividad física al menos 60 minutos diarios durante menos de 5 dias a la semana } \\
\hline Bogotá, D.C. & $76,7(73,4-80,1)$ & $84,5(80,9-88,2)$ & $81,2(79,0-83,4)(n=1.250)$ \\
\hline Bucaramanga & $75,8(73,1-78,6)$ & $83,1(79,3-86,9)$ & $79,8(77,4-82,1)(n=1.480)$ \\
\hline Cali & $75,9(71,1-80,7)$ & $81,5(77,4-85,7)$ & $79,0(75,6-82,4)(n=1.275)$ \\
\hline Manizales & $78,3(73,5-83,0)$ & $87,1(84,5-89,7)$ & $83,2(80,5-85,9)(n=1.346)$ \\
\hline Valledupar & $77,5(73,5-81,6$ & $81,8(79,2-84,3)$ & $81,8(79,2-84,3)(n=1.376)$ \\
\hline \multicolumn{4}{|c|}{ No caminar ni tomar cicla para ir y volver del colegio } \\
\hline Bogotá, D.C. & $52,1(43,8-60,5)$ & $47,6(39,6-55,7)$ & $49,4(42,2-56,7)(n=1.254)$ \\
\hline Bucaramanga & $50,7(46,9-54,5)$ & $54,1(49,9-58,3)$ & $52,5(49,2-55,9)(n=1.498)$ \\
\hline Cali & $53,0(48,4-57,5)$ & $51,8(43,9-59,7)$ & $52,3(46,6-58,0)(n=1.280)$ \\
\hline Manizales & $49,3(41,2-57,5)$ & $51,3(43,0-59,6)$ & $50,5(44,9-56,1)(n=1.359)$ \\
\hline Valledupar & $39,3(33,5-45,0)$ & $52,5(45,1-59,9)$ & $46,9(41,4-52,5)(n=1.384)$ \\
\hline \multicolumn{4}{|c|}{ Tres horas o más al día frente a la televisión o el computador } \\
\hline Bogotá, D.C. & $51,3(46,3-56,2)$ & $52,0(46,6-57,4)$ & $51,6(47,6-55,6)(n=1.247)$ \\
\hline Bucaramanga & $45,8(41,0-50,7)$ & $50,8(48,3-53,3)$ & $48,4(45,5-51,2)(n=1.496)$ \\
\hline Cali & $49,0(43,4-54,5)$ & $53,9(48,5-59,3)$ & $51,7(47,3-56,2)(n=1.289)$ \\
\hline Manizales & $51,2(47,5-54,9)$ & $59,8(53,8-65,8)$ & $56,0(51,3-60,7)(n=1.355)$ \\
\hline Valledupar & $38,2(33,8-42,6)$ & $47,8(41,6-53,9)$ & $43,8(39,5-48,2)(n=1.394)$ \\
\hline \multicolumn{4}{|c|}{ Cuatro o más clases semanales de educación física } \\
\hline Bogotá, D.C. & $21,3(16,1-26,4)$ & $23,5(19,5-27,6)$ & $22,5(18,7-26,2)(n=1.245)$ \\
\hline Bucaramanga & $28,2(22,6-33,8)$ & $23,2(18,2-28,1)$ & $25,5(21,0-29,9)(n=1.489)$ \\
\hline Cali & $33,6(29,0-38,2)$ & $29,7(25,3-34,1)$ & $31,4(27,7-35,1)(n=1.274)$ \\
\hline Manizales & $23,6(19,2-27,9)$ & $23,9(19,3-28,5)$ & $23,7(20,3-27,2)(n=1.351)$ \\
\hline Valledupar & $35,1(29,5-40,7)$ & $36,6(32,9-40,3)$ & $36,0(33,3-38,8)(n=1.376)$ \\
\hline
\end{tabular}

$\mathrm{Al}$ preguntar por el número de días que realizan actividad física, $80 \%$ de los estudiantes en las cinco ciudades reportaron que realizan actividad física 
al menos 60 minutos durante menos de cinco días a la semana. En Bogotá, Bucaramanga y Manizales las mujeres reportaron menores niveles de actividad física que los hombres, con diferencias que fueron significativas estadísticamente. Al comparar los resultados de hombres y de mujeres entre las distintas ciudades, no se observaron diferencias significativas (Tabla 2).

Actividad física para el transporte al colegio. Algo más de la mitad de los alumnos en las cinco ciudades $(50,3 \%)$ reportaron no caminar ni tomar una bicicleta para ir al colegio. El menor porcentaje fue en los hombres de Valledupar $(39,3 \%)$, con diferencias significativas al comparar con los hombres de Bucaramanga (50,7 \%) y Cali (53 \%). En Valledupar se observó una diferencia significativa entre hombres y mujeres en la proporción de estudiantes que reportaron no caminar o usar cicla para ir a estudiar: 39,3\% en hombres versus 52,5\% en mujeres (Tabla 2). En los colegios privados de Bogotá, 58,9\% (IC $95 \%$ 47,3 a 78,9) de los estudiantes de colegios privados reportaron que no realizaban actividad física para ir al colegio; en los colegios oficiales esta proporción fue de 45,9 \% (IC $95 \%$ 40,7 a 51,1).

Comportamiento sedentario en el tiempo libre. En conjunto en las cinco ciudades, 50,3\% de los estudiantes entre 13 y 15 años reportaron que gastaban tres horas o más diarias viendo TV, jugando en el computador o en una pantalla o hablando por teléfono. La ciudad donde los estudiantes reportaron el mayor nivel de tiempo dedicado a las pantallas fue Manizales (56\%), con diferencias significativas estadísticamente frente a Bucaramanga y Valledupar con porcentajes de $48,4 \%$ y 43,8 \%, respectivamente. En Valledupar se observaron los menores niveles de tiempo dedicado a la $\mathrm{TV}$ o al computador tanto en hombres $(38,2 \%)$ como en mujeres $(47,8 \%)$ (Tabla 2$)$.

Actividad física como parte del currículo escolar. En cuanto a las clases semanales de educación física, $27 \%$ de los estudiantes de ambos sexos en las cinco ciudades, manifestaron recibir cuatro o más clases semanales. La ciudad con mayor proporción en este indicador fue Valledupar (36\%), con diferencias que fueron significativas al comparar con Bogotá $(22,5 \%)$ y Manizales $(23,7 \%)$. No hubo diferencias significativas entre hombres y mujeres en ninguna ciudad (Tabla 2).

\section{DISCUSIÓN}

En general, los resultados mostraron bajos niveles de actividad física en los estudiantes de las cinco ciudades. Menos de $20 \%$ de los escolares entre 13 
y 15 años realizan actividad física durante 60 minutos diarios al menos cinco días a la semana y solamente $15 \%$ realizan actividad física diaria durante 7 días de la semana. Estos hallazgos son similares a los obtenidos en las respectivas encuestas EMSE realizadas en otros países latinoamericanos como Chile, Quito y Argentina (9). También son parecidos a los obtenidos en otras encuestas en jóvenes escolarizados en Brasil (12). La información de la encuesta del sistema de vigilancia de comportamientos de riesgo en jóvenes en los Estados Unidos, muestra que en ese país en 2007, 34,7\% de los adolescentes realizaron al menos 60 minutos de actividad física durante cinco o más días (13), lo que indica una mayor prevalencia de actividad física en los adolescentes de Estados Unidos.

En todas las ciudades los niveles de actividad física reportada fueron mayores en hombres que en mujeres. Esto es un hallazgo consistente en encuestas internacionales y nacionales sobre actividad física en escolares y que también ha sido reportado en adultos en Bogotá (13-15).

En relación con la actividad física para ir al colegio, las tres ciudades que reportaron mejores índices fueron Valledupar, Cali y Bucaramanga. Un hecho que podría explicar estos resultados es que las tres ciudades comparten características de clima cálido, lo que favorecería desplazarse a pie o en cicla para ir al colegio. De estas tres ciudades, Valledupar mostró los mejores índices, lo que adicionalmente podría deberse a ser una ciudad más pequeña que las otras dos. Otro factor que podría influir en los hallazgos es la seguridad percibida, que en Valledupar posiblemente sea mejor que en las demás ciudades. Sin embargo, no hay información suficiente para sustentar estas hipótesis. Ahora bien, estos resultados también deben ser tomados con cautela pues información, aun no publicada, de una investigación reciente en niños y adolescentes en Bogotá, mostró que en la categoría de "tomar una cicla para ir al colegio" se incluye cuando los jóvenes van sentados en el manubrio o en la parte de atrás de la cicla, que es manejada por uno de los padres o familiares (Sarmiento, Olga Lucía, Universidad de los Andes, Bogotá, comunicación verbal).

En Bogotá, aunque las diferencias no fueron significativas, los estudiantes de colegios oficiales reportaron mayores niveles de actividad física para el transporte al colegio. En general, los jóvenes que estudian en colegios oficiales pertenecen a estratos socioeconómico más bajos y en este sentido los resultados son comparables a un estudio brasileño que evidenció que los jóvenes de estratos más pobres caminan más para ir al colegio y por ende tienen mayores niveles de actividad física en el transporte (16). 
Al comparar los niveles de actividad física con la prevalencia de tabaquismo en estudiantes de las mismas ciudades (obtenida en la Encuesta Mundial de Tabaquismo en Jóvenes), se observó que los adolescentes de Valledupar y Bucaramanga, que presentan los mayores niveles de actividad física, muestran también las menores prevalencias de tabaquismo (17). Distintos estudios han mostrado que tanto en hombres y mujeres adolescentes, hay una asociación inversa entre la actividad física y el hábito de fumar, así como entre la actividad física y el uso de alcohol $(18,19)$.

En todas las ciudades más de la mitad de los alumnos de 13 a 15 años, reportaron que pasan tres horas o más al día, sentados viendo televisión, jugando en el computador o jugando videojuegos. Estos resultados son similares a los obtenidos en adolescentes de los Estados Unidos, donde 24,9 \% gastan tres horas o más diarias en videojuegos y 35,4 \% miran más de tres horas diarias de televisión (20). Aunque el presente estudio no permite analizar si aquellos jóvenes que son más activos físicamente, tienen menores índices de sedentarismo, algunos estudios han reportado que no hay una relación entre hacer deporte de manera activa y pasar menos tiempo ante la televisión (21).

En relación con las clases de educación física, la mayor prevalencia estuvo también en Valledupar, donde $36 \%$ de los estudiantes manifestaron recibir cuatro o más clases semanales. La práctica de actividad física como parte del currículo escolar ayuda a estudiantes que tienen un menor apoyo social para realizarla y se ha evidenciado que clases bien orientadas y planeadas logran una contribución significativa a cumplimiento de actividad moderada y vigorosa (22).

Una de las limitaciones de la presente encuesta es que aunque se hicieron pruebas de comprensión del español, la encuesta no ha sido validada para el país. La validación de encuestas similares realizadas en México ha mostrado que los adolescentes tienden a subestimar la actividad física intensa y el tiempo que pasan sentados y a sobreestimar la actividad física moderada (23).

Otra limitación la constituye el hecho de no haber tomado medidas antropométricas que permitan evaluar el índice de masa corporal y correlacionarlo con el nivel de actividad física. Las mediciones físicas conllevan más esfuerzos en términos del operativo, pero es una de las recomendaciones que se han hecho específicamente para esta encuesta y en general para la vigilancia de factores de riesgo con abordajes escalonados. 
Finalmente, las preguntas no permitieron diferenciar entre la actividad física en el tiempo libre y la actividad física para el transporte; cabe la posibilidad que los niveles de actividad física reportados incluyan también la actividad física realizada al transportarse al colegio.

A pesar de las anteriores limitaciones, los resultados presentados constituyen una línea de base muy importante para la toma de decisiones en el nivel local. En Bogotá, se ha estimado que un $5 \%$ de la mortalidad por enfermedades crónicas y un $2 \%$ de la mortalidad por todas las causas podrían prevenirse si la prevalencia de adultos inactivos físicamente se reduce (24). El espacio escolar constituye un escenario muy importante para lograr un impacto en este sentido.

Como estrategia de vigilancia de factores de riesgo, el presente estudio mostró altas tasas de respuesta escolar y estudiantil, que indican interés en la encuesta por parte de directores de colegios, padres de alumnos y alumnos. Una alta tasa de respuesta conlleva a una buena cobertura, que es una característica importante de cualquier sistema de vigilancia epidemiológica. La presente experiencia indica una alta posibilidad de implementar de forma periódica la EMSE por parte del Instituto Nacional de Cancerología

Agradecimientos: Los autores expresamos nuestros agradecimientos a cada uno de los estudiantes, profesores y rectores que participaron. También a Johanna Otero, a cada uno de los coordinadores y recolectores contratados por el Instituto Nacional de Cancerología y a las diferentes personas de los CDC y de la OPS que hicieron posible su realización. Finalmente queremos expresar un agradecimiento especial a John Duperly, quien revisó el manuscrito inicial e hizo anotaciones y comentarios de gran valor. Financiación: Este estudio fue realizado con recursos de inversión de la Nación, asignados al INC y recursos técnicos provenientes de la OPS y los CDC.

\section{REFERENCIAS}

1. Waxman A. Prevention of chronic diseases: WHO global strategy on diet, physical activity and health. Food Nutr Bull. 2003 Sep;24(3):281-4.

2. Warburton DE, Nicol CW, Bredin SS. Health benefits of physical activity: the evidence. CMAJ. 2006 Mar 14;174(6):801-9.

3. World Health Organization [Internet]. A European framework to promote physical activity for health. WHO Europe 2009; Disponible en http://www.euro.who.int/_data/assets/ pdf_file/0020/101684/E90191.pdf Consultado: Dic 212009.

4. US Department of Health \& Human Services. 2008 Physical Activity Guidelines for Americans. http://www health gov/US 2009 [cited 2009 Dec 3];Available from: URL: www.health.gov

5. Whitaker RC, Wright JA, Pepe MS, Seidel KD, Dietz WH. Predicting obesity in young adulthood from childhood and parental obesity. N Engl J Med. 1997 Sep 25;337(13):869-73. 
6. Hallal PC, Victora CG, Azevedo MR, Wells JC. Adolescent physical activity and health: a systematic review. Sports Med. 2006;36(12):1019-30.

7. Dehne KL, Riedner G. Adolescence--a dynamic concept. Reprod Health Matters. 2001 May;9(17):11-5.

8. Dobbins M, De CK, Robeson P, Husson H, Tirilis D. School-based physical activity programs for promoting physical activity and fitness in children and adolescents aged 6-18. Cochrane Database Syst Rev. 2009 Jan 21;(1):CD007651.

9. World Health Organization. Global School Based Student Health Survey (GSHS). WHO CHP 2009 [cited 2009 Nov 16];Available from: URL: http://www.who.int/chp/gshs/en/

10. Warren CW, Asma S, Lee J, Lea V, Mackay J. Global Tobacco Surveillance System: The GTSS Atlas. Atlanta: CDC Foundation; 2009.

11. Shah BVBBGBGS. Software for the Statistical Analysis of Correlated Data (SUDAAN): User's Manual release 7.5, 1997 (software documentation). Research Triangle Park, NC; 1997.

12. Bastos JP, Araujo CL, Hallal PC. Prevalence of insufficient physical activity and associated factors in Brazilian adolescents. J Phys Act Health 2008 Nov;5(6):777-94.

13. Li S, Treuth MS, Wang Y. How active are American adolescents and have they become less active? Obes Rev 2009 Oct 27.

14. Gomez LF, Duperly J, Lucumi DI, Gamez R, Venegas AS. Physical activity levels in adults living in Bogota (Colombia): prevalence and associated factors. Gac Sanit. 2005 May;19(3):206-13.

15. Gordia AP, de Quadros TM, de CW, Petroski EL. Adolescents' physical quality of life: associations with physical activity and sex. Rev Salud Publica (Bogota) 2009 Jan;11(1):50-61.

16. Hallal PC, Wells JC, Reichert FF, Anselmi L, Victora CG. Early determinants of physical activity in adolescence: prospective birth cohort study. BMJ. 2006 Apr 29;332 (7548):1002-7.

17. Pardo $C$, Piñeros $M$, Jones NR, Warren $C W$. Results of the global youth tobacco surveys in public schools in Bogota, Ciolombia. J Sch Health 2010; 80(3):141-5.

18. Charilaou M, Karekla M, Constantinou M, Price S. Relationship between physical activity and type of smoking behavior among adolescents and young adults in Cyprus. Nicotine Tob Res. 2009 Aug;11(8):969-76.

19. Korhonen T, Kujala UM, Rose RJ, Kaprio J. Physical activity in adolescence as a predictor of alcohol and illicit drug use in early adulthood: a longitudinal population-based twin study. Twin Res Hum Genet. 2009 Jun;12(3):261-8.

20. Eaton DK, Kann L, Kinchen S, Shanklin S, Ross J, Hawkins J, et al. Youth risk behavior surveillance-United States, 2007. MMWR Surveill Summ 2008 Jun 6;57(4):1-131.

21. Fernandes RA, Junior IF, Cardoso JR, Vaz Ronque ER, Loch MR, de Oliveira AR. Association between regular participation in sports and leisure time behaviors in Brazilian adolescents: a cross-sectional study. BMC Public Health. 2008 Sep 23;8:329.

22. Fairclough S, Stratton G. 'Physical education makes you fit and healthy'. Physical education's contribution to young people's physical activity levels. Health Educ Res. 2005 Feb;20(1):14-23.

23. Hernandez B, Gortmaker SL, Laird NM, Colditz GA, Parra-Cabrera S, Peterson KE. Validity and reproducibility of a questionnaire on physical activity and non-activity for school children in Mexico City. Salud Publica Mex. 2000 Jul;42(4):315-23.

24. Lobelo F, Pate R, Parra D, Duperly J, Pratt M. Burden of mortality associated to physical inactivity in Bogota, Colombia. Rev Salud Publica (Bogota). 2006 Dec;8 Suppl 2:28-41. 